\title{
Using wavefront coding technique as an optical encryption system: reliability analysis and vulnerabilities assessment
}

\author{
Mikhail V. Konnik \\ School of Electrical Engineering and Computer Science, \\ The University of Newcastle, Callaghan 2308 Australia
}

\begin{abstract}
Wavefront coding paradigm can be used not only for compensation of aberrations and depth-of-field improvement but also for an optical encryption. An optical convolution of the image with the PSF occurs when a diffractive optical element (DOE) with a known point spread function (PSF) is placed in the optical path. In this case, an optically encoded image is registered instead of the true image. Decoding of the registered image can be performed using standard digital deconvolution methods.

In such class of optical-digital systems, the PSF of the DOE is used as an encryption key. Therefore, a reliability and cryptographic resistance of such an encryption method depends on the size and complexity of the PSF used for optical encoding. This paper gives a preliminary analysis on reliability and possible vulnerabilities of such an encryption method. Experimental results on brute-force attack on the optically encrypted images are presented. Reliability estimation of optical coding based on wavefront coding paradigm is evaluated. An analysis of possible vulnerabilities is provided.
\end{abstract}

Keywords: optical encryption, coding vulnerabilities, reliability assessment

\section{INTRODUCTION}

The optical encryption systems can be roughly divided into mainly optical and mainly digital systems. Optical encryption techniques, such as lensless systems, ${ }^{1}$ 4-f based systems, ${ }^{2}$ fractional Fourier transform ${ }^{3}$ or systems based on the double random phase mask, ${ }^{4}$ use high parallelism of optical images processing to provide fast and secure encryption. Digital systems, on the other hand, are performing all operations in numerical form ${ }^{5}$ and therefore have greater flexibility. Among the digital encryption systems, Virtual Optics system ${ }^{6}$ and virtualoptical-holography ${ }^{7}$ are noteworthy.

The proposed hybrid optical-digital encryption system ${ }^{8}$ based on the wavefront coding paradigm ${ }^{9}$ attempts to use the strength of both optical (high speed and parallelism) and digital systems (simple implementation on a computer). The diffractive optical element (DOE) with a known point spread function (PSF) can be used as an encryption key. An optical convolution of the image (e.g., printed on a paper and placed as an input scene in front of the system) with the PSF of the DOE can be though as a result of an encryption of the input scene. In this case, optically encoded images are registered by the digital camera instead of the original image. Decryption is performed using standard digital deconvolution methods and the image of the DOE PSF as a decryption key. The process of optical encryption is fast and secure, and the resulting image can be transferred via open communication channels.

The characteristics of the PSF of the DOE, which is used as an encryption key, influence the reliability and cryptographic resistance of such an encryption method. This contribution discusses the cryptographic strength and vulnerabilities of the proposed optical-digital encryption technique. The vulnerability assessment in this paper is generally based on computational complexity, which can then be expressed in computational time needed to find the encryption key. The experimental results include the possible experimental scheme for the plain-text attack, the tolerance to rotation and scaling of the decryption key, and the methods of reduce the complexity of the brute-force attack on the encrypted image with binarization of decryption key images.

Further author information: Mikhail V. Konnik, mail@mvkonnik.info 


\section{EXPERIMENTAL SETUP FOR OPTICAL ENCRYPTION AND DIGITAL DECRYPTION}

The hybrid optical-digital encryption system based on wavefront coding paradigm performs the encryption of the input scene optically (optical convolution of the DOE's PSF and the input scene) and the digital decryption (using deconvolution algorithms).

Experimental setup with optical encryption is shown in Fig. 1. The ND:YAG laser radiation 1 is attenuated by a polariser 2 and focused on the micro lens 3. To make the monochromatical illumination spatially uniform, the spatial frequency filter 4 is used. The spatial coherence of laser light is destroyed by a rotating ground glass 5 to reduce the influence of speckle noise on the encoding process. The light reflected from the input scene 6 is recorded by a digital camera 7. The DOE (kinoform) 8 is placed inside the camera space between the lens and the photosensor. The computer 9 controls the process of capturing images and can perform the decoding of images.

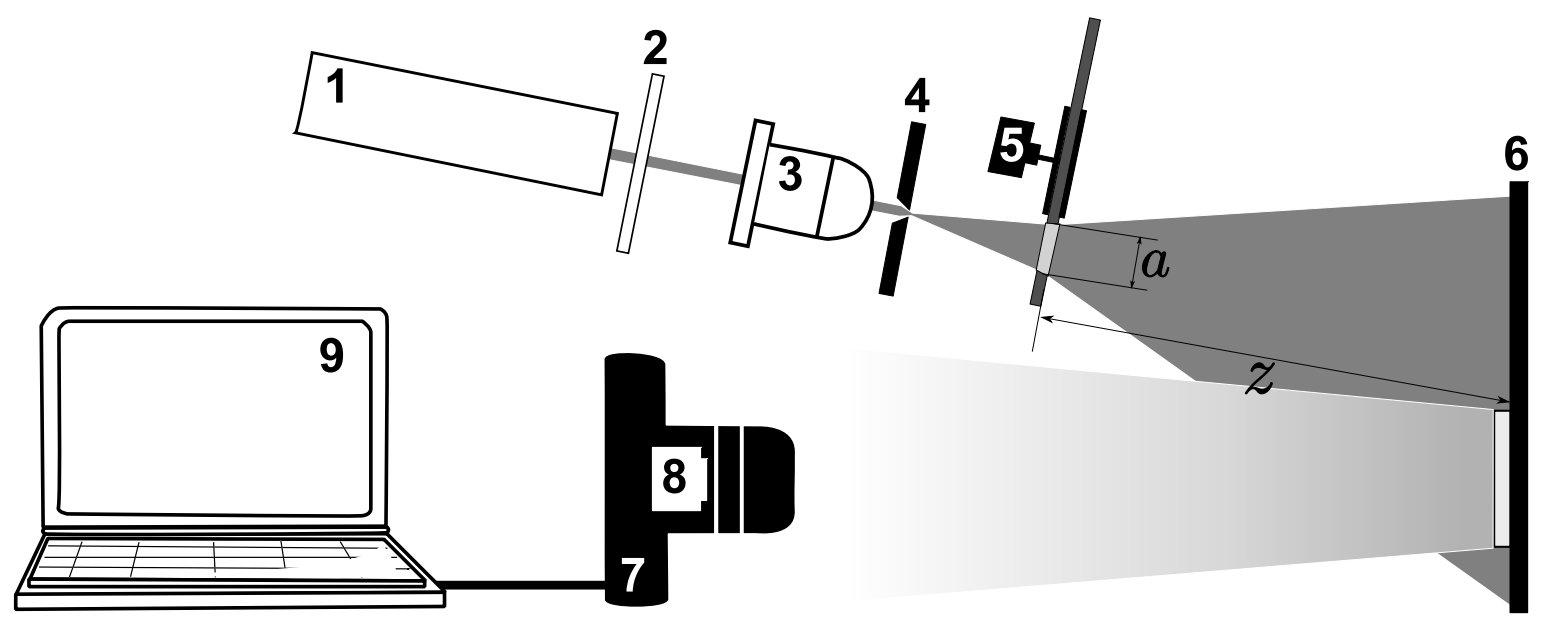

Figure 1. Experimental setup of optical encryption: 1 - YAG:Nd laser with KDP, 2 - attenuation filter, 3 - micro lens, 4 - spatial frequency filter, 5 - rotating ground glass for the destruction of spatial coherence of a laser light, 6 - input scene with the message being encrypted, 7 - digital camera, 8 - DOE (kinoform), 9 - computer.

The optical encryption of the input scene is performed as follows. The convolution of the source image (Fig. 2a) and the PSF (Fig 2b) of the synthesised DOE (kinoform in this work) produces the encrypted image (Fig. 2c). It worth to mention that the PSF's of the DOE will scale depending on the used wavelength of the illumination, because the DOE contains a synthesised phase mask. This can be though as an additional security feature: the PSF image registered in red light will be of different scale than the one registered in blue light. Such circumstance can be used to make the plain-text attack more difficult (see Section 4.1).

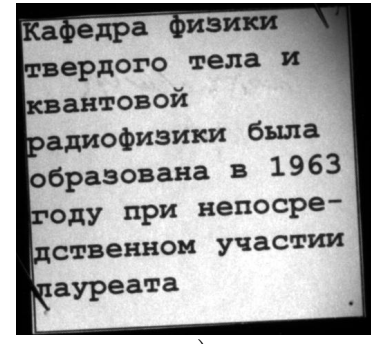

a)

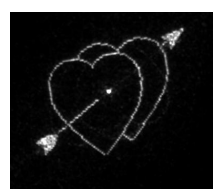

b)

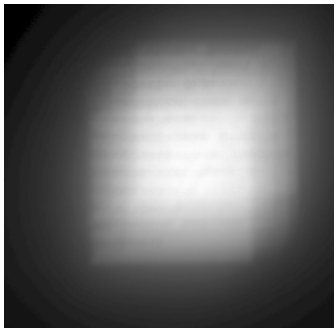

c)

Figure 2. The process of optical encoding: a) original image, b) encoding PSF, a) coded image.

The decryption is performed using standard digital deconvolution methods ${ }^{10}$ such as Wiener (one-step) or Richardson-Lucy (iterative). The digital convolution of the encrypted image (see Fig. 3a) and the decoding PSF 
(Fig. 3b, that is inverse with respect to the encoding PSF shown in Fig.2b) is performed. The inverse PSF is calculated by the deconvolution algorithm from the image of the encryption PSF. If the key is correct, then the image after the deconvolution is readable again (see Fig. 3c).

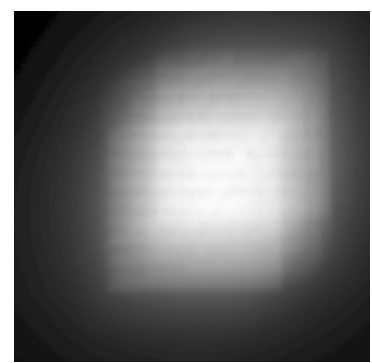

a)

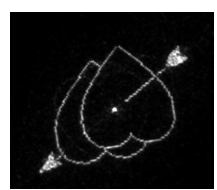

b)

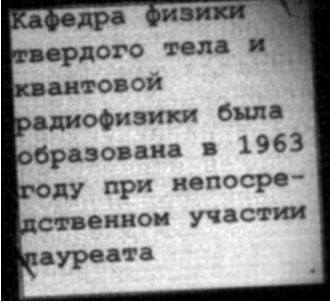

c)

Figure 3. The process of optical decoding: a) encrypted image, b) decoding PSF, a) decoded image.

As can be seen from the example above, the image is restored with minimal distortion. Typical normalised root mean squared error (NRMSE) for the reconstructed images from the original is of order 0.2-0.3 (corresponding to $20-30 \%$ ), which corresponds to a small level of distortions and artefacts on the image.

\section{ASSESSMENT OF THE CRYPTOGRAPHIC STRENGTH OF THE OPTICAL-DIGITAL ENCRYPTION METHOD}

To estimate the number of computational operations and the time required for exhaustive search of the decryption PSF, we assume that each multiplication operation requires one floating-point operation (addition operation does not take into account). Consider all the images are of size that is powers of two in order to use the maximum speed of FFT. We also do not take into account the analysis time of the decoded image, as well as the time required to generate the decoding PSF.

Consider the digital image $h(x, y)$ of the coding PSF is of size $M \cdot N$ samples and $K$ halftones. In case of brute-force attack, all possible combinations of the PSF can be estimated as $V_{t h e o r}=K^{M \cdot N}$ variants. Let the size of the encoded digital image $g(x, y)$ is $A \cdot B$ samples. Typical image size of the encrypted image can exceed $2000 \cdot 3000$ pixels. A brute-force attack of the optically encoded image will require the following operations:

1. computation of the Fourier transform $H(u, v)$ from the PSF $h(x, y)$ of size $M \cdot N$ (at least $O_{F F T}=$ $M N \cdot \log _{2}(M \cdot N)$ operation required);

2. evaluation of the inverse (decoding) PSF using the image deconvolution algorithm (at least $O_{\text {iOTF }}=$ $4 *(A \cdot B)^{2}$ operation required);

3. computation of the Fourier transform $G(u, v)$ from the encoded image $g(x, y)$ of size $A \cdot B$;

4. multiplication of the Fourier transforms of the PSF decoder $Y(u, v)$ and the encoded image $G(u, v)$ size $A \cdot B$ to decode the image:

5. calculation of the inverse Fourier transform of the decoded image $F(u, v)$ size $A \cdot B$ (at least $O_{i F F T}=$ $A B \cdot \log _{2}(A \cdot B)$ operations $)$.

Thus, the total number of operations to brute force can be estimated as:

$$
C_{B F . \text { theor. }}=\left[M N \cdot \log _{2}(M \cdot N)+4 \cdot(A \cdot B)^{2}+A B \cdot \log _{2}(A \cdot B)\right] \cdot K^{(M \cdot N)}
$$

To quantify the number of operations, we assume that the encoded image is of size $A=2048, B=2048$ pixels, the encoding PSF has $K=16$ halftones and $M=300, N=300$ pixels. The number of options for different PSF is $V_{\text {theor.optenc }}=16^{300 \cdot 300} \approx 10^{108370}$.

The procedure above can be shortened in order to reduce the number of computational operations. Nevertheless, it is clear that the brute-force attack on the optically encoded image requires significant computing resources. In this case the attacker is facing the following difficulties: 
1. estimation the size of the coding PSF $(M \cdot N)$ to analyse the encrypted image (and reduce the computational burden);

2. evaluation of halftones $K$ of the encoding PSF;

3. deconvolution of each encrypted image requires considerable computation time;

4. there is no definite criterion of successful decryption - each attempt will generate some image, which must be analysed separately (that is difficult to automate).

However, the number of brute-force attacks can be reduced. According to the experimental data, the decoding of encrypted images may be accomplished by binary PSF image even if the true image was encrypted with halftone PSF. Using the binarized image of the decoding PSF reduces the computational time. Although the decoded images by binarized PSF are of inferior quality, the attacker will be able to read (at least in part) the encrypted message.

Other potential weaknesses of the optical-digital encryption method include: presentation of specially generated images to the encryption system to obtain an encryption key. ${ }^{11,12}$ Despite the fact that these attacks can be effective, they are unlikely because they require either access to the system of coding, or at least to the encoding DOE. In the following sections these options are discussed and an analysis of the attacks is presented.

\section{RESULTS ON THE BRUTE-FORCE ATTACK OF THE OPTICALLY ENCRYPTED IMAGE}

There are several possible ways to compromise this optical encryption process. We assume that all that the adversary has is the digital file with the encrypted message. Therefore, there are the following cases of the decryption with the mismatched PSF (encryption key) to consider:

1. the image decoding PSF is scaled;

2. the scale of decoding PSF is correct but the image of PSF is rotated;

3. the image of the decoding PSF is binarized (in order to reduce the computational burden in brute-force attack).

Such variants of an attack to the proposed optical encryption technique ${ }^{8}$ are discussed in the following sections.

\subsection{Plain-Text attack on the encrypted image}

One of the simplest methods to attack the system is to use the plain-text attacks. If the adversary has access to the encryption device or to the encryption key (diffractive optical element, DOE) then it is possible to recover the encryption PSF by feeding specially formed signals to the system. For example, using Dirac's delta function (that can be implemented as a bright point source), one can recover encryption PSF of the DOE and use it to decrypt the images. An example of the experimental setup that can be used to compromise the system is provided on Fig. 4.
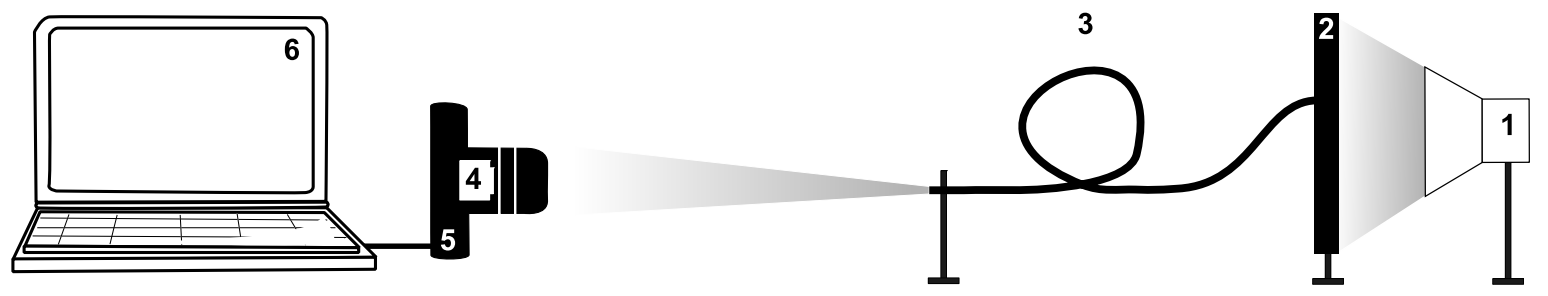

Figure 4. The experimental setup for the registration of the DOE PSF: 1 - light source, 2 - opaque optical waveguide mount, 3 - optical waveguide, 4 - DOE (kinoform), 5 - digital camera, 6 - computer. 
The optical scheme in Fig. 4 consists of a light source 1 with the same wavelength as used for the encryption, which is used to form a point light source by opaque optical waveguide mount 2 and the optical waveguide 3 . The convolution of the PSF of the DOE 4 (encryption key) is registered by the digital camera 5 and stored as an image file on the computer 6 . As a result, the image of the encryption key (DOE's PSF, see Fig. 2b) will be registered and further unauthorised decryption of the coded images can be performed.

Therefore, due to the nature of convolution, it is possible to compromise this encryption method using bright point source or a small bright area on the black background.

\subsection{Attack on the encrypted image with the rotated or scaled decoding PSF}

This example illustrates the recovery of the encoded image (see Fig. 5 a) when using the decoding PSF that is rotated (see Fig. 5 b) or proportionally scaled (see Fig. 5 c).

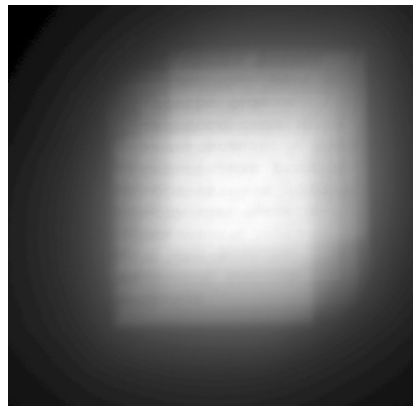

a)

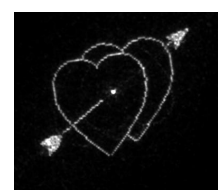

b)

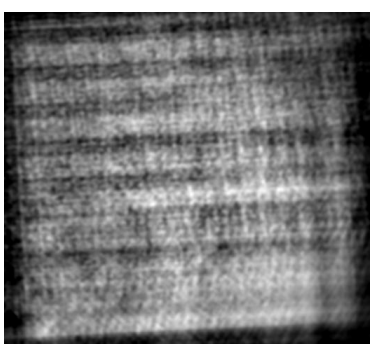

e)

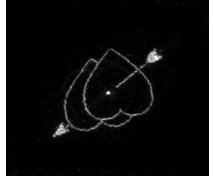

c)

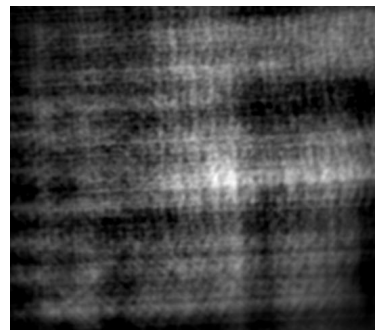

f)

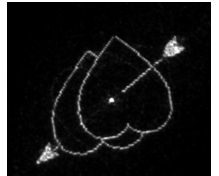

d)

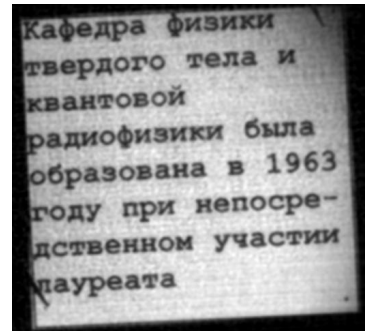

j)

Figure 5. Recovery of optically encoded images: a) the original encoded image, and b) the unscaled PSF rotated by $180^{\circ}$, c) the PSF scaled by $140 \%, \mathrm{~d})$ the correct decrypting PSF, e) the image decrypted with the PSF rotated by $180^{\circ}$, f) the image decrypted with the PSF scaled by $140 \%$, and j) the decryption with the correct PSF.

As can be seen from the results in Fig. 5, the image decrypted with the rotated or scaled PSF (see Fig. 5b and Fig. 5c) can not be read (see Fig. 5e and Fig. 5f, respectively). However, the structure of the encryption message can be revealed (at least in part) to give to an adversary clue of the encryption key. The results allow saying that the decryption of coded images using the distorted PSF (either inaccurately recorded or found by brute-force) in the following cases:

1. the rotation of the PSF is not more than $\pm 3^{\circ}$;

2. proportional scaling of the PSF is not more than $\pm 20 \%$.

These requirements will provide the normalised root mean square error (NRMSE) that is less than 50\% compared with the decryption of the image with the known PSF. This means that if the correct decoding PSF is rotated on more than $\pm 3^{\circ}$ or scaled to more than $\pm 20 \%$, the NRMSE of the decrypted image is too high and the decrypted message cannot be identified.

\subsection{Attack on the encrypted image with the binarized or thinned-out decoding PSF}

Instead of halftone decoding PSF, one can use binarized PSF for the cryptographic brute-force attack on the encoded image to significantly reduce computational burden. Binarization reduces the number of halftones in the PSF to $K=2$, as shown in Fig. 6b and Fig. 6c. Thus, instead of computing all $K^{M \cdot N}$ variants, one can compute only $2^{(M \cdot N)}$ PSFs. 
In this example, when the PSF is of size $M=300, N=300$ pixels, binarization reduces the number of options for different PSF with $V_{\text {theor.optenc }}=K^{M \cdot N}=16^{300 \cdot 300} \approx 10^{108370}$ to $V_{\text {pract.bin.optenc }}=2^{(M \cdot N)}=$ $2^{300 \cdot 300} \approx 10^{27090}$. However, it is significantly more difficult analysis of the resulting decoded image, because the reconstructed images are of low contrast and visual quality (see Fig. 6e and f).

Images of the PSF were binarized by the threshold from 50 to 225 digital numbers (DN) (out of $256 \mathrm{DN}, 8$ bit images) in increments of 25 DN. For each binarized PSF, the number of significant pixels that differ from the background was determined. Next, the encoded image (see Fig. 6a) was restored by binarized PSF (i.e., with a loss of significant pixels of PSF such as on Fig. $6 \mathrm{~b}$ and c).

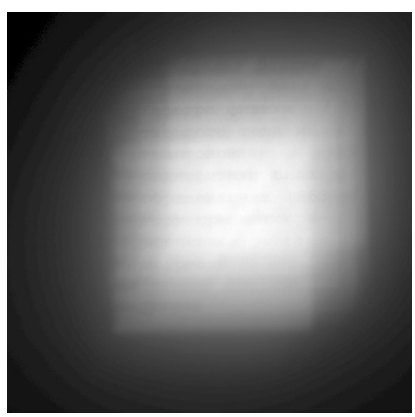

a)

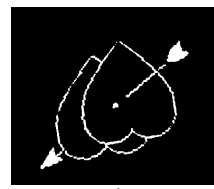

b)

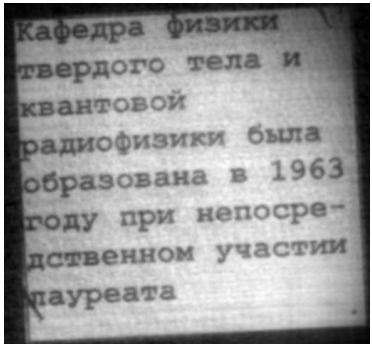

e)

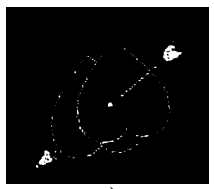

c)

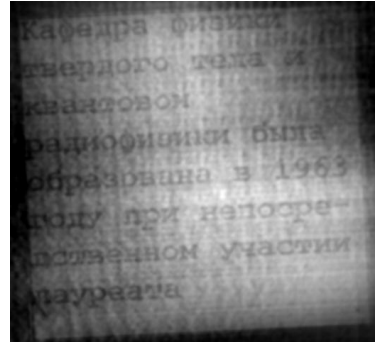

f)

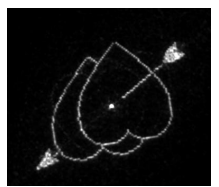

d)

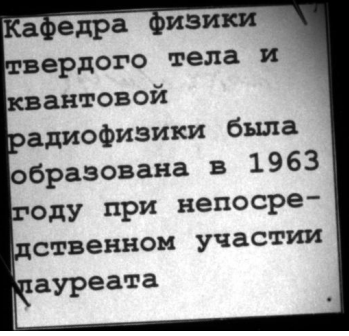

j)

Figure 6. Digital restoration of optically encoded images using the binarized PSF: a) the original encoded image, b) binarization threshold for the PSF is 75 out of 256 DN, c) binarization threshold for PSF is 150 out of 256 DN, d) correct decryption PSF without binarization, e) decryption with the binarized PSF of threshold 75 DN, f) decryption with the binarized PSF of threshold $150 \mathrm{DN}$, and j) the decryption with the correct decryption PSF.

Thus, using visual and quantitative evaluation, it was possible to determine the threshold level for the binarization of the PSF and the corresponding number of significant elements of the PSF. The images decoded with lower threshold (even with the correct decoding PSF) will be of poor visual quality and there impossible to identify. The results for several typical encryption PSFs are summarised in Table. 1 where each PSF is represented by one string in the table.

Table 1. Determination of the number of significant pixels in the encoding PSF.

\begin{tabular}{llllll}
\hline $\begin{array}{l}\text { PSF } \\
\text { number }\end{array}$ & $\begin{array}{l}\text { Total number } \\
\text { of pixels }\end{array}$ & $\begin{array}{l}\text { Non-zero } \\
\text { (coding) } \\
\text { pixels }\end{array}$ & $\begin{array}{l}\text { Minimal pixels in PSF } \\
\text { to identify the de- } \\
\text { coded image }\end{array}$ & $\begin{array}{l}\text { RMS from the } \\
\text { original }\end{array}$ & $\begin{array}{l}\text { Maximum loss } \\
\text { of the significant } \\
\text { pixels in PSF }\end{array}$ \\
\hline PSF 1 & 117648 & 8929 & 2134 & 0.26 & $76 \%$ \\
PSF 2 & 67496 & 2560 & 1022 & 0.23 & $60 \%$ \\
PSF 3 & 130082 & 5489 & 1599 & 0.34 & $70 \%$ \\
\hline
\end{tabular}

The data from the Table. 1 can be interpreted as follows. The registered image of the PSF is of size $342 \times 344=117648$, which has 8929 non-zero pixels that actually used for the optical encryption. If the adversary wants to brute-force attack the encrypted image with a number of generated PSFs, it is reasonable to generate binary images to reduce the computational efforts. The data in Table. 1 show that the encrypted image can be decoded (although the quality of the image will be poor, with the NRMS error of about 0.3 from the original) even when about $70 \%$ of significant pixels are lost. In other words, the attacker can read the encrypted message if the generated PSF contains about 30\%. This is a price to pay for the convenience of the optical encryption based on the optical convolution. 


\section{RESULTS DISCUSSION}

The results in the previous sections show the vulnerabilities of the optical-digital encryption method based on wavefront-coding paradigm. The method is obviously vulnerable to the plain-text attack, when the attacker uses specific known images to compromise an encryption system. Due to the nature of a convolution, it is possible to compromise this method using bright point source or a small bright area on the black background.

To summarise the experimental results of the cryptographic vulnerabilities of the optical-digital encryption system based on wavefront coding, we can note that:

1. the number of halftones on the DOE's PSF has little effect on the encryption strength of the method (the encrypted image can be decrypted with correct binarized PSF, although the quality of the image will be lower);

2. removing up to $70 \%$ of the non-zero pixels in the decoding PSF still produces the readable decrypted image (but reduces the visual quality of the image);

3. rotation of the decryption PSF on $\pm 3^{\circ}$ or proportional scaling of the PSF for $\pm 20 \%$ is maximum allowable distortions for the image to be decrypted in case when the coding PSF is known exactly.

Based on this, we can estimate feasibility of the brute-force attacks (see Eq. 1) if only encrypted image is known.

\subsection{Feasibility of a brute force attack}

Binarization of the image of the decoding PSF can significantly reduce the number of operations required for the brute-force attack from $V_{\text {theor.optenc }}=K^{M \cdot N}$ to $V_{\text {pract.bin.group.optenc }}=2^{(M \cdot N) / 4}$, where $K$ is the number of halftones of the encryption PSF and $M, N$ is height and width* of the image of the coding PSF. For the typical encryption PSFs used in our work (PSF image size is $300 \times 300$ pixels) this means that binarization allows to reduce the number of variants from $V_{\text {theor.optenc }}=16^{300 \times 300} \approx 10^{108370}$ down to $V_{\text {pract.bin.optenc }}=2^{(300 \times 300)} \approx 10^{27090}$, and with pixels grouping - to $V_{\text {pract.bin.group.optenc }}=2^{(300 \times 300) / 4} \approx 10^{6770}$. Those estimations, however, can be considered as a worst-case scenario. Even then, the number of the variants is still large and such optical-digital encryption can be safely used for a short-term encryption.

On the other side, the difficulty of the analysis of the decrypted images is significant. This is due to the fact that every decryption attempt produces some image, and it is difficult to automate the process of the analysis. Furthermore, since the encryption is done with the real hardware, noise and distortions add another level of complexity for the attacker.

\subsection{Comparison with Double Random Phase Encryption}

It is worth to compare the proposed hybrid optical-digital encryption method based on wavefront coding with other methods, for example with Double Random Phase Encryption (DRPE). Although DRPE is more cryptographically resistant, several attacks are proposed against the DRPE scheme.

Similarly to the our proposed method, the DRPE is found to be vulnerable to the plain-text attacks ${ }^{13}$ using Dirac's delta function or uniform (spatially constant) image. With at most three chosen plain-ciphered image pairs, it is possible to recover the two encryption keys and break the system. However, the data in ${ }^{13}$ is only a theoretical review. Also, there is no quantitative analysis of decryptability: only "fuzzy" visual estimations are presented.

More wise attack is the use of approximate version of the phase mask, especially to binary phase mask. Binarization of the phase mask reduces possible combinations dramatically. In order to reduce the combinations of decryption keys further it is advisable ${ }^{11,12}$ to decode the image with partial window of second key. The same is true for our method: approximate image of the decoding PSF (binarized on some threshold) can be used to reduce the number of variants of generated PSF necessary to decode the image.

More detailed analysis of attacks on phase encoding and quantisation influence is covered in. ${ }^{14,15}$ An iterative attempt to decrypt DRPE images is proposed in. ${ }^{16}$ Further analysis of the DRPE scheme can be found in papers. ${ }^{11,17,18}$

${ }^{*}$ As above, the size of the PSF image depends on the wavelength of the illumination used for the encryption. In this work we use green laser $\lambda=535 \mathrm{~nm}$ Nd:YAG with frequency doubling. 


\section{CONCLUSION}

This contribution addresses the problem of the reliability and potential vulnerabilities of the hybrid opticaldigital encryption system based on the wavefront coding. The idea of the method is to use diffractive optical element DOE (kinoform) to optically perform a convolution of the DOE's PSF (encryption key) with the image of the input scene (a text or an image printed on the paper) illuminated by monochromatical light source. The decryption is performed digitally using standard deconvolution algorithms, with the initial image of the PSF as a decryption key.

The strong points of the proposed method are speed of the encryption, security (the registered image has been already encrypted), and a relatively simple and compact hardware implementation (digital camera, kinoform and computer). The system, however, has vulnerabilities that can reduce its cryptographic resistance and decrease the reliability of the encrypted images:

1. vulnerability to the plain-text attacks: if the attacker has an access to the encryption device or to encryption key (kinoform, DOE) then it is possible to recover the encryption PSF using specially formed signals as the encryption system's input (Dirac delta function);

2. the decryption key (the image of the coding PSF of the DOE) is not unique: changing or removing the non-zero pixels in the decoding PSF still produces a readable decrypted image;

3. rotation of the decryption PSF on $\pm 3^{\circ}$ or proportional scaling of the PSF for $\pm 20 \%$ is maximum allowable distortions for the image to be decrypted in case when the coding PSF is known exactly;

4. binarization of the encryption PSF can be used by an attacker to reduce the variants of generated PSFs significantly.

Despite of those vulnerabilities of the proposed optical-digital encryption system, the method can be used for short-term encryption of text messages or military orders printed on a paper, or for the encryption of archive sensible printed materials. The number of variants for brute-force attack and difficulties of the analysis are significant enough to provide reliable and portable encryption hardware for military or intelligence needs.

\section{REFERENCES}

[1] Situ, G. and Zhang, J., "A lensless optical security system based on computer-generated phase only masks," Optics Communications 232, 115-122 (Mar. 2004).

[2] Wang, X., Zhao, D., and Chen, L., "Image encryption based on extended fractional fourier transform and digital holography technique," Optics Communications 260, 449-453 (Apr. 2006).

[3] Nishchal, N. K., Joseph, J., and Singh, K., "Fully phase-encrypted memory using cascaded extended fractional fourier transform," Optics and Lasers in Engineering 42, 141-151 (Aug. 2004).

[4] Enrique Tajahuerce, Osamu Matoba, S. C. V. and Javidi, B., "Optoelectronic information encryption with phase-shifting interferometry," Applied Optics Vol. 39, No. 14, 2313-2320 (10 May 2000).

[5] Benbasat, A. Y., "A survey of current optical security techniques," tech. rep., MIT Media Lab, Prepared for Prof. Cardinal Warde 6.637 Spring 1999 Research Project (April 15, 1999).

[6] Peng, X., Cui, Z., and Tan, T., "Information encryption with virtual-optics imaging system," Optics Communications 212, 235-245 (Nov. 2002).

[7] Xiang Peng and Peng Zhang, "Security of virtual-optics-based cryptosystem," Optik 117, 525-531 (2006).

[8] Konnik, M. V., "Hybrid optical-digital encryption system based on wavefront coding paradigm," in [paper 8398-25 in Proceedings of SPIE Defense, Security, and Sensing Symposium, Baltimore, Maryland, USA], (2012 (to appear)).

[9] Edward R. Dowski, J. and Johnson, G. E., "Wavefront coding: a modern method of achieving highperformance and/or low-cost imaging systems," in [Proc. SPIE, Current Developments in Optical Design and Optical Engineering VIII], 3779(1), 137-145 (1999). 
[10] Sergey N. Starikov, Mikhail V. Konnik, Edward A. Manykin, and Vladislav G. Rodin, "Linear methods for input scenes restoration from signals of optical-digital pattern recognition correlator," in [Proceedings of SPIE, Defence and Security Symposium, Optical Pattern Recognition XX, Vol. 7340:73400B], (2009).

[11] A. Carnicer, M. Montes-Usategui, S. Arcos, and I. Juvells, "Vulnerability to chosen-cyphertext attacks of optical encryption schemes based on double random phase keys," Optics Letters 30, 1644-1646 (2005).

[12] X. Peng, H. Wei, and P. Zhang, "Chosen-plaintext attack on lensless double-random phase encoding in the fresnel domain," Optics Letters 31, 3261-3263 (2006).

[13] Yann Frauel, Albertina Castro, Thomas J. Naughton, and Bahram Javidi, "Resistance of the double random phase encryption against various attacks," Optics Express Vol. 15, No. 16, 10253-10265 (6 August 2007).

[14] David S. Monaghan, Guohai Situ, Unnikrishnan Gopinathan, Thomas J. Naughton, and John T. Sheridan, "Role of phase key in the double random phase encoding technique: an error analysis," Applied Optics Vol. 47, No. 21, 3808-3816 (20 July 2008).

[15] David S. Monaghan, Unnikrishnan Gopinathan, Thomas J. Naughton, and John T. Sheridan, "Key-space analysis of double random phase encryption technique," Applied Optics Vol. 46, No. 26, 6641-6647 (10 September 2007).

[16] Guohai Situ, Unnikrishnan Gopinathan, David S. Monaghan, and John T. Sheridan, "Cryptanalysis of optical security systems with significant output images," Applied Optics Vol. 46, No. 22, 5257-5262 (1 August 2007).

[17] Unnikrishnan Gopinathan, David S. Monaghan, T. J. N. and Sheridan, J. T., "A known-plaintext heuristic attack on the fourier plane encryption algorithm," Optics Express Vol. 14, No. 8, 3181-3186 (2006).

[18] X. Peng, P. Zhang, H. Wei, and B. Yu, "Known-plaintext attack on optical encryption based on double random phase keys," Optics Letters 31, 1044-1046 (2006). 